Pak. j. sci. ind. res. Ser. A: phys. sci. 201457 (2) 63-69

\title{
Dynamics of Electron Concentration for Ionospheric Region of Pakistan
}

\author{
Syed Nazeer Alam ${ }^{a *}$ and Muhammad Ayub Khan Yousufzai ${ }^{b}$ \\ ${ }^{a}$ Department of Electronics \& Power Engineering, Navy Engineering College, \\ National University of Sciences \& Technology Karachi, Pakistan \\ ${ }^{b}$ Department of Applied Physics, Solar-Terrestrial \& Atmospheric Research Wing and Institute of \\ Space \& Planetary Astrophysics, University of Karachi, Karachi-75270, Pakistan
}

(received February 13, 2013; revised June 2, 2013; accepted June 19, 2013)

\begin{abstract}
The fluctuating dynamics of electron density is highly dependent on altitude from center of the earth. The long distance communication via $\mathrm{F}_{2}$ layer is the best suited through refraction of radio wave in the range of 3-30 MHz. In present study, the $\mathrm{F}_{2}$ layer hourly data for 2006, recorded at SUPARCO Islamabad Ionosphere Station (SIIS), located at latitude $33.75^{\circ} \mathrm{N}$ and longitude $72.87^{\circ} \mathrm{E}$ have been considered. The recorded ordinary wave frequency has been utilised to compute relationship with variation in electron concentration. The estimation of variability is determined for forecast and modeling purposes. The standard techniques have been performed such as regression, stochastic analysis and parameter estimation using data obtained from source. Predicting sky wave propagation at Pakistan ionospheric region has been presented.
\end{abstract}

Keywords: ionosphere, ordinary wave frequency, total electron count, electron density, temporal variations

\section{Introduction}

Our atmosphere is divided into several regions: the troposphere, the stratosphere and the ionosphere above the sea-level. The ionosphere resembles an optical device that reflects and refracts the radio waves. This process depends on the degree of ionisation of the ionosphere. This region is comprised of ionised structure with varying electron-ion concentration highly dependent upon solar radiation. It lies between altitudes of 70 to $600 \mathrm{~km}$ and participate in radio propagation. The ionosphere tends to be stratified, rather than regular, in its distribution. The existence of ionosphere as an electronically conducting region had been postulated in 1883 to explain the daily and seasonal variations in the geomagnetic field in 1902, appropriately that ionosphere contains free electrons and ions produced by solar ionising radiation (Robert and Andrew, 2004). The two reflecting layers in the ionosphere 90 to 110 $\mathrm{Km}$ called Heaviside layer (E region) and Appleton layer is $240 \mathrm{Km}$ (Harrison, 1958). The ionosphere structure (C, D, E and F layers) characterised by level of ionisation depends upon strength of ultraviolet (UV), alpha and gama radiations from the sun and cosmic rays. The ionisation is the greatest in summer and day

*Author for correspondence; E-mail: nazeer@pnec.nust.edu.pk time, least in the winter and night. The sunspot, a standard index of solar activity has influence on the radio flux density of ionosphere (Barclay, 2003). The Appleton layer is about four times as strong a reflector as the Heaviside layer owing to the stronger concentration of electrons and ions (Harrison, 1958). It is this layer, which makes possible propagation of short waves round the world. In case of oblique-incidence ionospheric soundings the signal can be propagated via single hop, as is in present case or through successive reflections of the waves from the earth-ionosphere waveguide walls (Afanasiev et al., 2001).

The layer. All ionosphere layers vary in altitude and density according to the solar cycles but these variations do not always have the sense in the different layers. The F layer extends $100 \mathrm{Km}$ to $300 \mathrm{Km}$ in night above the earth's surface. The bifurcation of $\mathrm{F}$ layer into $\mathrm{F}_{1}$ and $\mathrm{F}_{2}$ is observed by low noon value, evening concentration of ionisation and slow electron disappearance after sunset (Nazeer and Yousufzai, 2013). The $\mathrm{F}_{1}$ layer is located at an altitude $150 \mathrm{Km}$ to $210 \mathrm{Km}$ and is called Chapman layer. This presents a regular stratification at moderate latitudes. Its maximum electron density $\left(N_{e}\right)$ is given by equation (1), where $R_{0}$ is sunspot number (Armal, 1974). 


$$
\mathrm{N}_{\mathrm{e}}=2.5 * 10^{11}\left(1+0.0062 \mathrm{R}_{\mathrm{o}}\right)
$$

The altitude of the $\mathrm{F}_{2}$ layer is at about $300 \mathrm{Km}$, its behaviour is different, since it exhibits seasonal anomaly from other layers and well supported for long distance ionosphere communication. The electron concentration varies between $20 * 10^{11} \mathrm{e} / \mathrm{m}^{3}$ during the day and $5 * 10^{11} \mathrm{e} / \mathrm{m}^{3}$ during the night. In fact the total electron concentration (TEC) in $\mathrm{F}_{2}$ layer is $>F_{1}$ layer due to (i) smaller electron loss and (ii) variation in solar radiation (Dolukhanov, 1971).

\section{Materials and Methods}

Long distance propagation of electromagnetic waves for frequencies less than $30 \mathrm{MHz}$ is controlled by the ionosphere. Martyn's theory suggests its principal characteristics (Armal, 1974). The ionosphere study has been carried out using radio methods by ground stations located at Islamabad, Pakistan. The mathematical model strategy has been implemented to compute electron concentration $\left(\mathrm{N}_{\mathrm{e}}\right)$ as a result of temporal and seasonal variation in refractive index $(\mu)$ for recorded hourly ordinary wave frequencies of $\mathrm{F}_{2}$ layer for 2006 using Digisond DG-256 equipment at Islamabad station. To evaluate relationship between ionosphere parameters statistical technique has been implemented to study parametric variability using Statistica and Minitab tools.

Ordinary wave frequency. The critical frequency is the limiting frequency at or below which a radio wave is reflected by an ionosphere layer using oblique incidence. The highest frequency returned to earth, when radiated upward in the vertical direction. Its varying value is dependent on electron-ion measurement round the clock. For $F_{2}$ layer the ordinary wave frequency $\left(f_{o}\right)$ is related to peak value of electron concentration (Henry and Owen, 1969) given in equation (2).

$$
\mathrm{f}_{\mathrm{o}}=\sqrt{80.8 \mathrm{~N}}_{\mathrm{e}} \cong 9 \sqrt{\mathrm{N}_{\mathrm{e}}(\max ) \cdots}
$$

For the 244 samples recorded hourly for year 2006, the maximum and minimum ordinary wave frequency are 9.49 $\mathrm{MHz}$ and 3.16 MHz, respectively. The reflection and refraction from the $\mathrm{F}_{2}$ layer is the major factor in HF communications.

Electron density. The uv, $\alpha$ and $\gamma$ emission have a greater ionising power, when the radiation frequency is higher. The density of electrons in the ionosphere varies as a function of geomagnetic latitude, diurnal cycle, annual cycle, and sunspot cycle. The peak value of electron density of $\mathrm{F}_{2}$ layer is calculated with respect to measured ordinary critical frequency as given in the expression, equation (3) postulated by Anderson and Matsushita in 1974 (Robert and Andrew, 2004; Barclay, 2003).

$$
\mathrm{N}_{\mathrm{e}}(\max )=1.24 * 10^{4} \mathrm{f}_{\mathrm{o}}^{2}(\mathrm{MHz}) \mathrm{e} / \mathrm{cm}^{3}
$$

The calculated electron density maximum value is $1.1167 * 10^{12}$ and minimum value is $1.2382 * 10^{11} \mathrm{e} / \mathrm{m}^{3}$, respectively, with mean of $3.4274 * 10^{11}$ having standard deviation of $1.2871 \mathrm{e} / \mathrm{m}^{3}$. King et al. (1964) calculated refractive index and absorption coefficient of weakly ionised air and showed that with the increase of ion density, the refractive index decreases. The gradual decrease in refractive index from layer to layer causes the upward obliquely incident ray to gradually bend away from the normal and ultimately return to the earth after total refraction (Ghosh, 1998). The ionosphere is weak plasma. Larmor (1924) suggested that plasma possesses dielectric constant $>$ unity. The established relationship to compute value of refractive index of ionosphere layers ignoring influence of magnetic field is mentioned in equation (4) and (5).

$$
\begin{aligned}
\mu^{2} & =1-\left[\frac{\omega_{p}}{\omega}\right]^{2} \\
f_{p} & =\sqrt{\frac{N_{e}^{2}}{\pi m \varepsilon_{o}}}
\end{aligned}
$$

where;

$\omega_{\mathrm{p}}$ and $\omega$ are plasma frequency and frequency of the wave, mass of electron $(\mathrm{m})$, with $\varepsilon_{0}$, medium characteristic constants (Ghosh, 1998). The total refraction occurs when the collision frequency of the ionosphere is less than the radio frequency and if the electron density in the ionosphere is great enough.

In present study, the variation in the computed values of refractive index is 0.94823 to 0.95226 with mean 0.95007 and a least standard deviation of 0.00059 offering bending of EM wave from $\mathrm{F}_{2}$ layer at day and night finally providing skip distance mean value of $4099.5 \mathrm{Km}$ with standard deviation of $357.4 \mathrm{Km}$ and refraction of waves from layers of varying altitude.

Exploratory data analytic approach. The exploratory data analytic approach (EDAA) plays an important role 
in statistical analysis. EDA approach relies heavily on graphical methods i.e., scheming of histogram for representation of distribution with single quantitative variable. The continuous envelope provides the hypothetical limit of samples. In this particular study, the sampling distribution of critical frequency and electron concentration are presented in histogram plots shown in Fig. 1-2. The distribution shows slight right

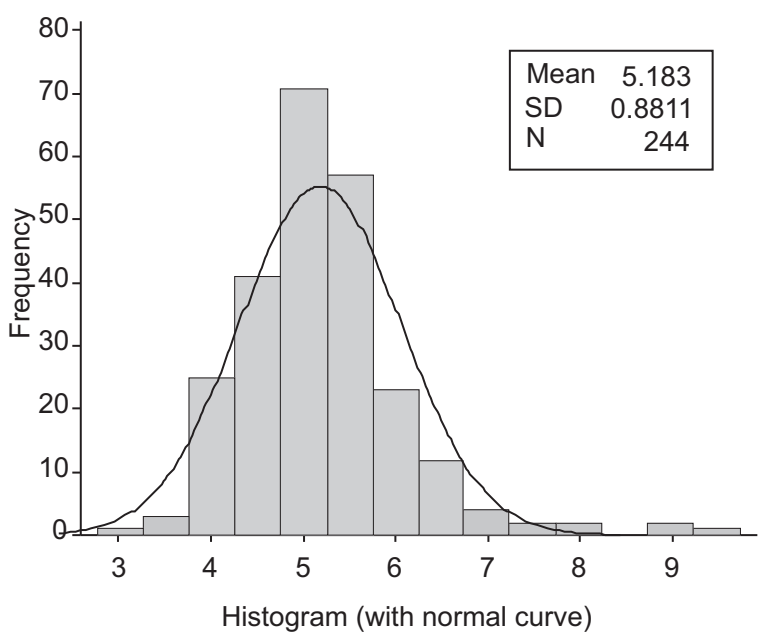

Fig. 1. Distribution of observed ordinary wave frequency.

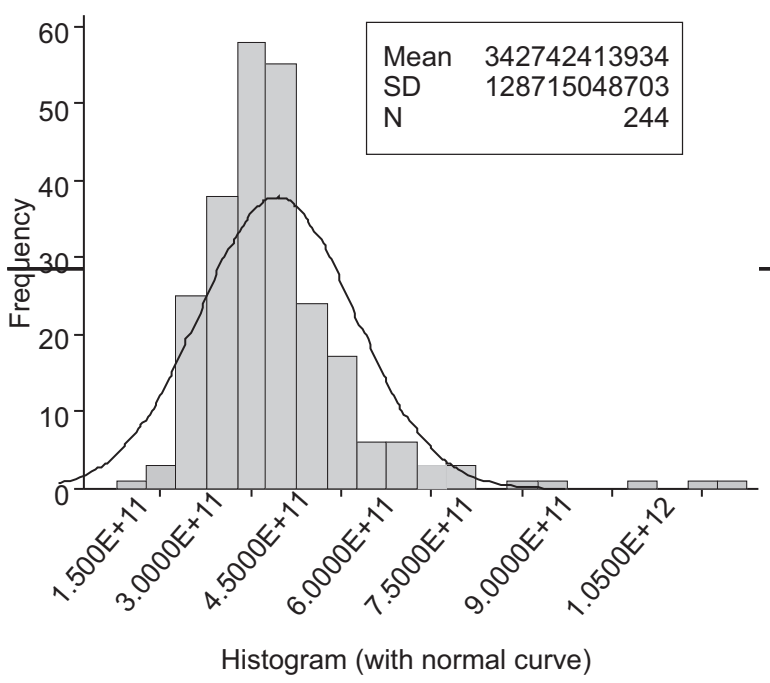

Fig. 2. Distribution of computed electron concentration. scenes with few outliers those can be minimised in the later studies using robustness. The coefficient of variation $\left(\mathrm{C}_{\mathrm{v}}\right)$, a normalised measure of dispersion of probability distribution is defined as ratio of standard deviation to the mean. From computed statistical data, $\mathrm{C}_{\mathrm{v}}=0.17$ and 0.37 for ordinary wave frequency and electron concentration, respectively, show distribution with low variance i.e., $\mathrm{C}_{\mathrm{v}}<1$. The corresponding signal-to-noise ratio, inverse of $\mathrm{C}_{\mathrm{v}}$ determined as 5.88 and 2.66. The other important statistical values are presented in Table 1.

Regression analytic strategy. According to Sir Francis Galton worked quantifying the strength of relationship to estimate or predict the known values of one variable with other variable is given by Walpole et al. (2012). The bivariate analysis presented in scatter plot in Fig. 3(a-b) displays positive correlation between ordinary wave critical frequency and electron concentration for linear and quadratic fit models of $\mathrm{F}^{2}$ layer. The linear regression depicts line having an intercept equal - $4.05 * 10^{11}$ with slope of $1.44 * 10^{11}$ shows fairly fits through our sample data consisting of 244 observations. The Pearson's statistic, coefficient of determination, $\mathrm{R}^{2}=0.9750, \mathrm{R}^{2}$ (adj) $=97.5 \%, \mathrm{R}^{2}$ (pred) $=97.26 \%$ and Durbin-Watson statistic of 1.27183 . The

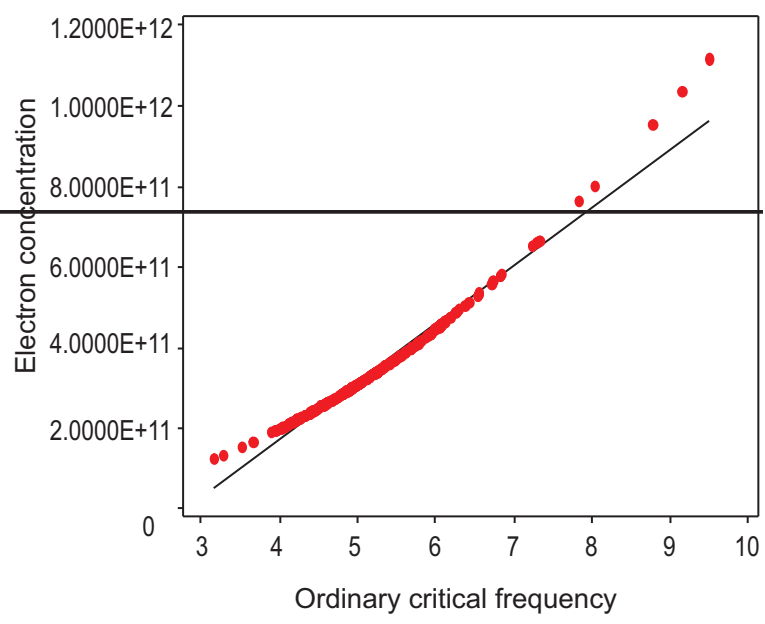

Fig. 3(a). Scatter plot for ordinary wave frequency and electron concentration.

Table 1. Computed parameter values

\begin{tabular}{llllllll}
\hline \hline Parameter & Trim mean & Median & Variance & IQR & Skewness & Kurtosis & P value \\
\hline $\mathrm{f}_{\mathrm{o}}$ & 5.12 & 5.07 & 0.7763 & 0.9290 & 1.45 & 4.60 & 0.000 \\
$\mathrm{~N}_{\mathrm{e}}$ & $3.29 * 10^{11}$ & $3.19 * 10^{11}$ & $1.65 * 10^{22}$ & $1.17 * 10^{11}$ & 2.52 & 10.52 & 0.000 \\
\hline \hline
\end{tabular}




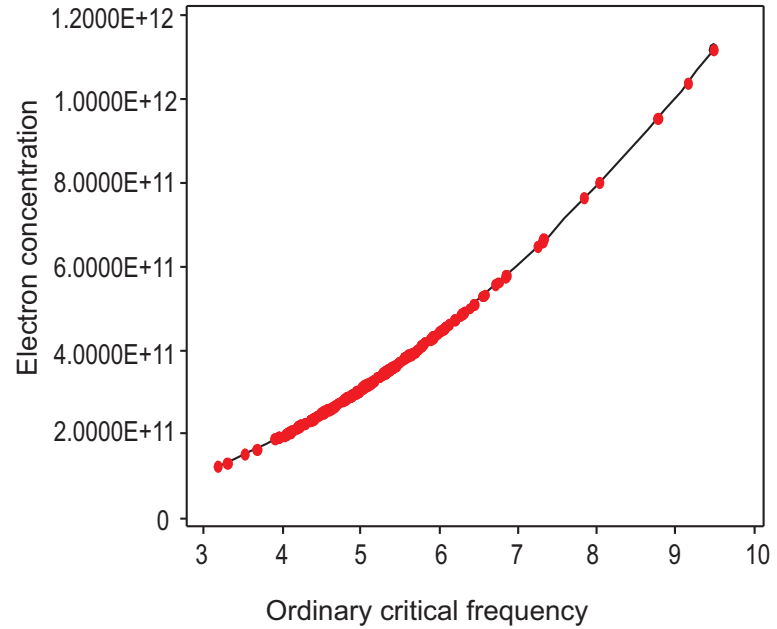

Fig. 3(b). Scatter plot for ordinary wave frequency and electron concentration.

linear fit regression model equation is mentioned in equation (6).

$$
\mathrm{N}_{\mathrm{e}}=-4.05 * 10^{11}+1.44 * 10^{11} \mathrm{f}_{\mathrm{o}}
$$

The quadratic model incorporating all the values suggest the best fit with $=97.55 \%$. The quadratic fit regression model equation is mentioned in equation (7).

$\mathrm{N}_{\mathrm{e}}=2.1847 * 10^{6}-8.763 * 10^{5} \mathrm{f}_{\mathrm{o}}+1.24 * 10^{10} \mathrm{f}_{\mathrm{o}}^{2}$

These results show strong relationship i.e., changes in ordinary wave critical frequency are in accordance and relevant to real time astrophysics variations in ionosphere (Allan and Richard, 1985).

Stochastic approach. The stochastic approach suggesting correlation between adjacent points in time is the best investigation in terms of dependence of the present and the past values (Diebold, 1998). In this study seasonal pattern with periodic behaviour of variation in ordinary wave frequency and electron concentration against time with the best data fit in case of both linear and quadratic are shown in Fig. 4(a-b) and $5(\mathrm{a}-\mathrm{b})$, respectively. The time plots show variations reflecting increase and decrease of data in the ionosphere of $\mathrm{F}^{2}$ layer in day and $\mathrm{F}$ layer during night at Islamabad ionosphere region.

The simplest exponential characterisation method is single exponential smoothing (SES). This method, when properly applied, reveals more clearly the underlying trend, seasonal, cyclic components and non-stationarity

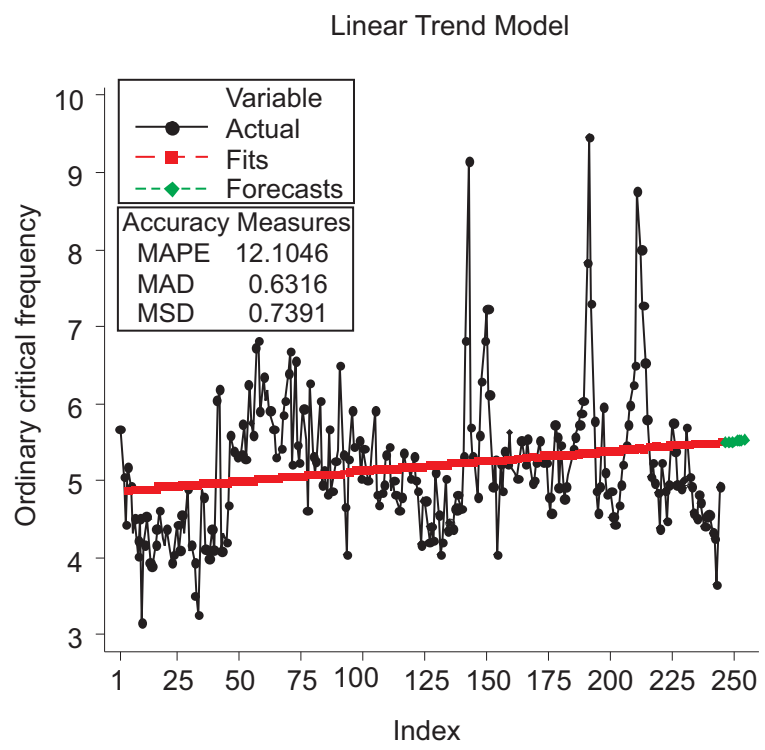

Fig. 4(a). Time plot of ordinary wave frequency.

Quadratic Trend Model

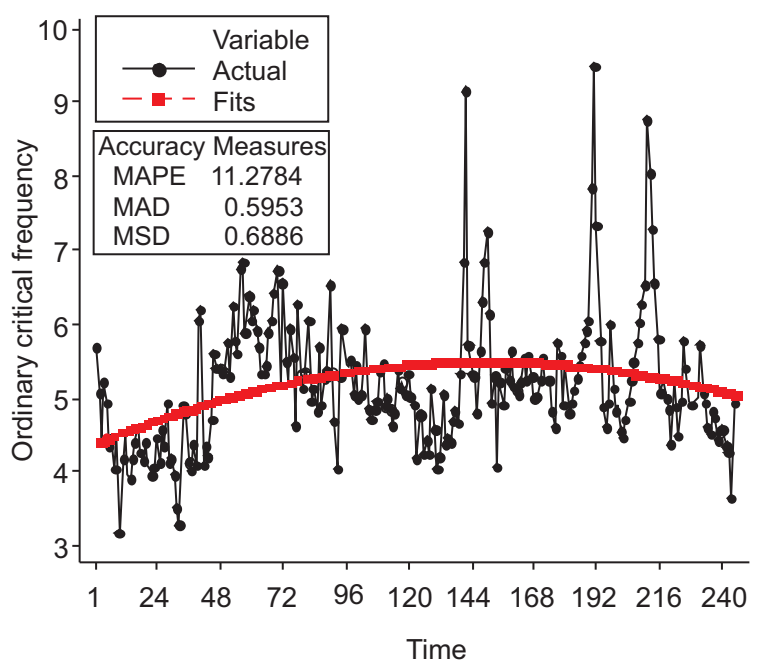

Fig. 4(b). Time plot of ordinary wave frequency.

present in time series plots are removed by differencing the data or by fitting some type of trend curve. The stochastic plots indicated in Fig. 4-5 exhibit rising trend for electron concentration and ordinary wave frequency under investigation. These plots witness a linear fit is sufficient to remove the trend but the variance (amplitude) is still varying with time. The single smoothing forecasting expression is mentioned in Equation (8). The forecast plots along with actual time series and single exponential smoothed series for fitting with linear trend curve for both parameters are shown 


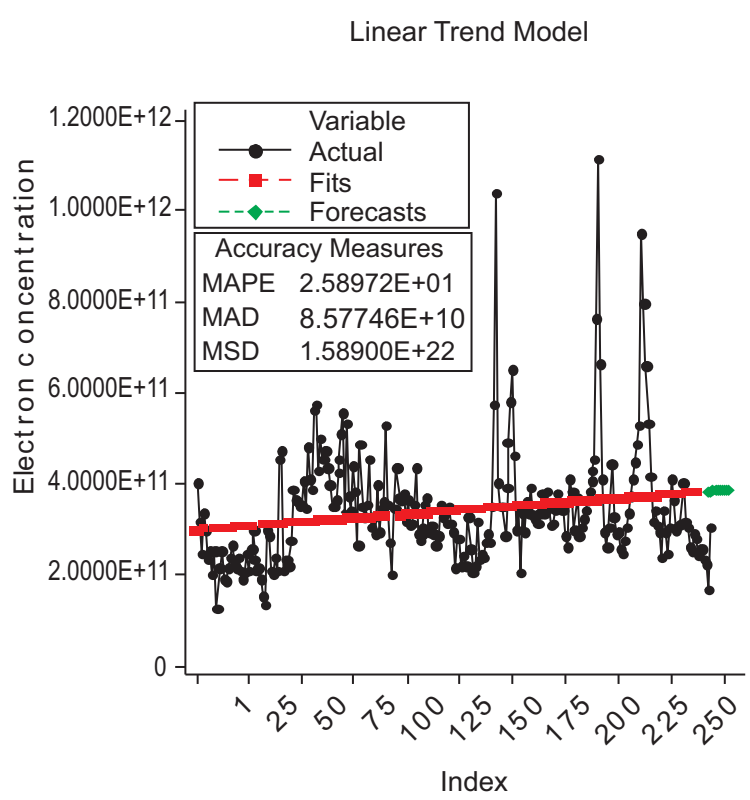

Fig. 5(a). Time plot of electron frequency.

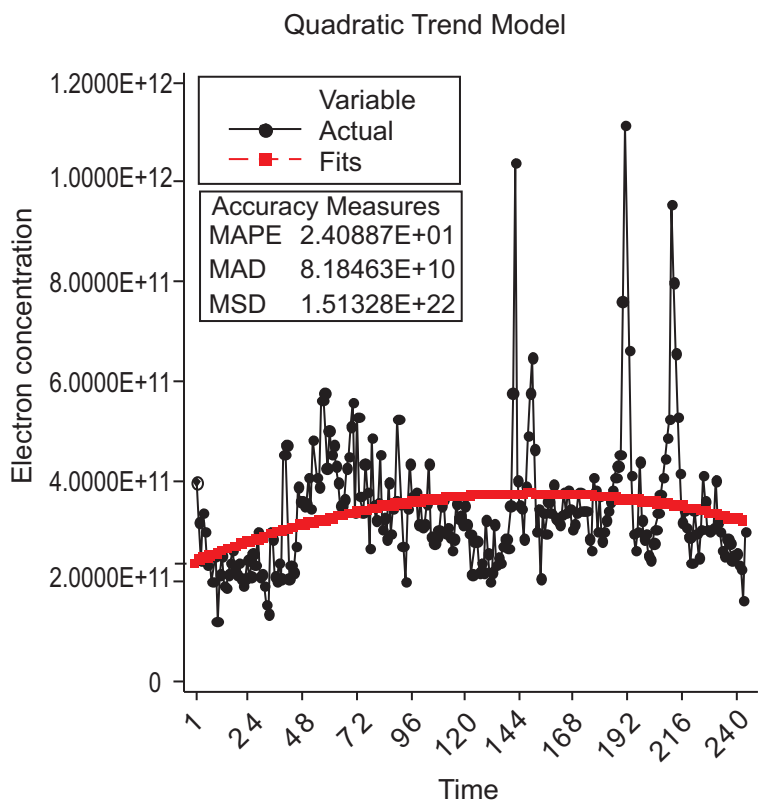

Fig. 5(b). Time plot of electron concentration.

separately (Walpole et al., 2012) in Fig. 6-7. In our case a $95 \%$ confidence interval will contain the true value of the population parameter with probability $95 \%$.

$$
\begin{aligned}
& \mathrm{F}_{\mathrm{t}+1}=\alpha \mathrm{Y}_{\mathrm{t}}+(1-\alpha) \mathrm{F}_{\mathrm{t}} \\
& 0<\alpha \leq 1 \text { for } \mathrm{t}>0
\end{aligned}
$$

The single smoothing coefficient, $\alpha=0.718347$ and 0.824733 explain level of smoothing for mean percentage

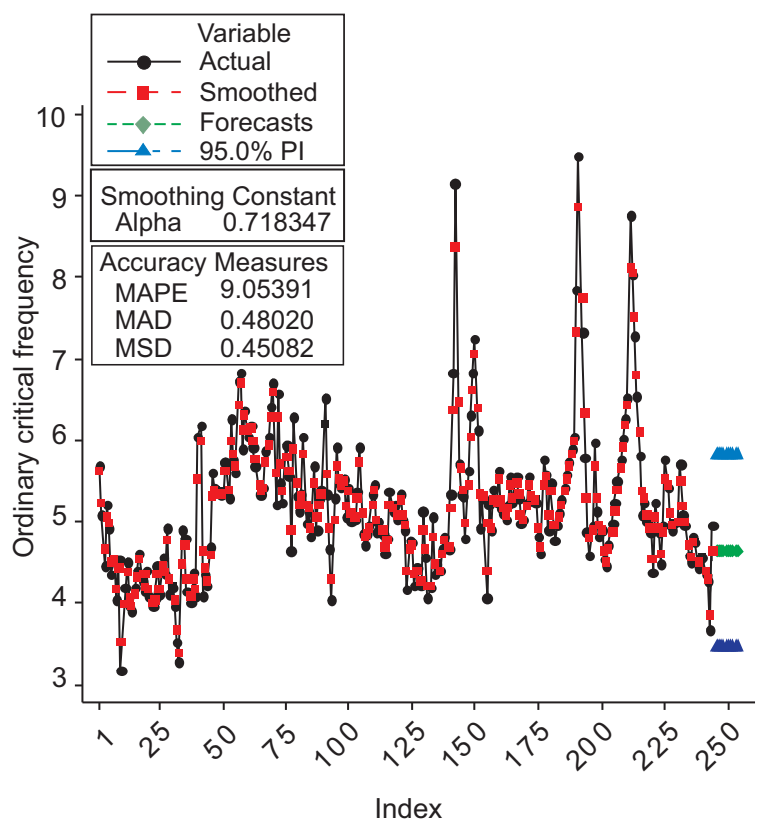

Fig. 6. Ordinary wave frequency-actual and exponential smoothing values.

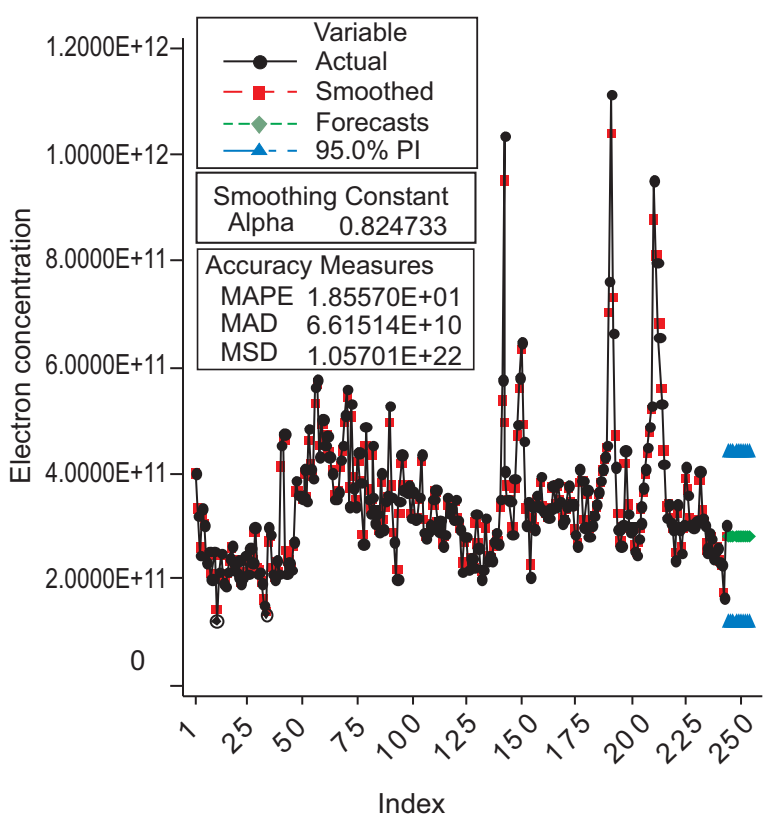

Fig. 7. Electron concentration-actual and exponential smoothing values.

error (MPE), mean absolute percentage error (MAPE) witness degree of accuracy in quantitative forecast with least residual with $95 \%$ prediction interval and how responsive the model is to show fluctuation in the data. This could be illustrated using ordinary wave critical frequency and electron concentration (Makridakis et al., 1983). The summary of errors for both parameters is 
mentioned in the expressions inside the illustration.

$$
\mathrm{F}_{\mathrm{t}+1}=\mathrm{F}_{\mathrm{t}}+\propto\left(\mathrm{X}_{\mathrm{t}}-\mathrm{F}_{\mathrm{t}}\right)=\mathrm{F}_{\mathrm{t}}+\alpha\left(\mathrm{e}_{\mathrm{t}}\right)
$$

The forecast values for ordinary wave frequency and electron concentration are computed using Bootstrapping technique, which estimates the distribution of an estimate by generating a large number of samples with replacement from the sample and re-computing the estimate for each of them (Ricardo et al., 2006) mentioned in model equation (10).

$$
\begin{aligned}
\mathrm{F}_{\mathrm{t}+1} & =\alpha \mathrm{Y}_{\text {origin }}+(1-\alpha) \mathrm{F}_{\mathrm{t}} \\
\mathrm{F}_{245} & =(0.718347)(4.94)+(1-0.718347)(4.63078) \\
& =4.853 \mathrm{MHz} \\
\mathrm{F}_{245} & =(0.824733)\left(3.022605 * 10^{11}\right)+(1-0.824733) \\
& \left(2.80586 * 10^{11}\right) \\
= & 2.987 * 10^{11} \mathrm{e} / \mathrm{m}^{3}
\end{aligned}
$$

Table 2. Forecast values obtained from bootstrap technique

\begin{tabular}{lll}
\hline \hline $\begin{array}{l}\text { Sample } \\
\text { no }\end{array}$ & $\begin{array}{l}\mathrm{f}_{\mathrm{o}} \\
\mathrm{MHz}\end{array}$ & $\begin{array}{l}\mathrm{N}_{\mathrm{e}} \\
\mathrm{e} / \mathrm{m}^{3}\end{array}$ \\
\hline 245 & 4.853 & $2.987 * 10^{11}$ \\
246 & 4.915 & $3.018 * 10^{11}$ \\
247 & 4.933 & $3.024 * 10^{11}$ \\
248 & 4.933 & $3.025 * 10^{11}$ \\
249 & 4.938 & $3.025 * 10^{11}$ \\
\hline
\end{tabular}

\section{Results and Discussion}

In this study physical mechanism of electron-ion production in the ionosphere have been discussed and quantified. The changeability observed in electron concentration is due to solar radiations received by the ionosphere. The presence of vertically distributed layered ionosphere structure, its temporal behaviour cause deviation in wave path and effect on radio wave communication have been analysed and values are predicted using boot strap smoothing approach. The data demonstrate evidence of parameter's fluctuating dynamic over period of time and season at low latitude ionosphere medium. To obtain results Digisound-256 hourly recorded data of ordinary wave frequency provided to compute the other parameters. The techniques implemented have been performed and the values of parameters are determined. The data distribution and trends have been found appropriate leading to the physical interpretation of the process occurred in the ionosphere. The forecast values have been obtained from the models developed for ionosphere region of Pakistan. These parametric values are useful for public, government and private sector organisations.

\section{Acknowledgement}

The authors are grateful to the officials of Pakistan Space \& Upper Atmosphere Research Commission (SUPARCO) for providing ionosphere data. These data helped us in carrying out this research work.

\section{References}

Afanasiev, N.T., Grozov, V.P., Nosov, V.E., Tinin, M.V. 2001. On the chirp-signal structure along the oblique-incidence ionospherric sounding path. Journal of Atmosphere and Solar-Terrestrial Physics, 63: 11-16.

Allan, H.M., Richard, W.K. 1985. Probability, Statistics, and Decision Making in the Atmospheric Sciences, pp. 301-304, Westview Press, Michigan, USA.

Armal, P. 1974. Radio Wave Propagation, pp. 122-123, Macmillan Press, Ltd., UK.

Barclay, L.W. 2003. Propagation of Radio Waves, pp. $160-168,2^{\text {nd }}$ edition, Institution of Electrical Engineers, London, UK.

Diebold, F.X. 1998. Elements of Forecasting, pp. 4-77, South-Western College Publication, USA.

Dolukhanov, M. 1971. Propagation of Radio Waves, pp. 214-215, translated by B.V., Kuznetsov, Mir Publisher, Moscow, Russia.

Durbin, J., Watson, G.S. 1951. Testing of serial correlation in least square regression, II. Biometrika, 38: 159-179.

Ghosh, S.N. 1998. Electromagnetic Theory and Wave Propagation, pp. 118-119, Narosa Publishing House, New Delhi, India.

Harrison, J.A. 1958. The Story of the Ionosphere; or, Exploring with Wireless Waves, pp. 62-65, Hulton Educational Publications, London, UK.

Henry, R., Owen, K. 1969. Introduction to Ionospheric Physics, Academic Press, Inc, Library of Congress No. 69-12280 111 Fifth Avenue, New York, 10003, USA.

King, J.W., Smith, P.A., Eccles, D., Fooks, G.F., Helm, H. 1964. Preliminary investigation of the structure of the upper atmosphere as observed by the topside sounder satellite, alouette. Proceeding of the Royal Society London, Series A, 281: 464-487. 
Larmor, J. 1924. Why wireless electric rays can bend round the earth. Nature, 114: 650-651.

Makridakis, S., Wheelwright, S.C., Victor, E.M. 1983. Forecasting: Methods and Applications, pp. 8493; 355-356, $2^{\text {nd }}$ edition, John Wiley \& Sons, New York, USA.

Nazeer, A., Yousuzai, A. 2013. Study the stochastic perception of refractive index variability of ionosphere, International Journal of Scientific \& Engineering Research, 4: 217-221.

Ricardo, A. M., Martin, R.D., Victor, J. Y. 2006. Robust
Statistics: Theory and Methods, pp. 140-141, John Wiley \& Son Ltd., England, UK.

Robert, W.S., Andrew, F.N. 2004. Ionospheres Physics, Plasma Physics, and Chemistry, (Cambridge Atmospheric and Space Science Series), 570 pp., Cambridge University Press, Cambridge, UK.

Walpole, R.E., Myers, R.H., Myers, S.L., Ye, K.E. 2012. Probability \& Statistics for Engineers \& Scientists, 816 pp., $9^{\text {th }}$ edition, Pearson Education International, Boston, USA. 